\title{
Multi-scale First-Principles Modeling of Three-Phase System of Polymer Electrolyte Membrane Fuel Cell
}

\author{
Giuseppe F. Brunello ${ }^{a}$, Ji Il Choi ${ }^{a}$, David Harvey ${ }^{b}$ and Seung Soon Jang a,c \\ a School of Materials Science and Engineering, Georgia Institute of Technology,771 \\ Ferst Drive NW, Atlanta, GA 30332-0245, USA; ${ }^{\mathrm{b}}$ Ballard Power Systems, 9000 Glenlyon \\ Parkway, Burnaby, BC V5J 5J8, Canada
}

The three-phase system consisting of Nafion, graphite and platinum in the presence of water is studied using molecule dynamics simulation. The force fields describing the molecular interaction between the components in the system are developed to reproduce the energies calculated from density functional theory modeling. The configuration of such complicated three-phase system is predicted through MD simulations. The nanophasesegregation and transport properties are investigated from the equilibrium state. The coverage of the electrolyte on the platinum surface and the dissolution of oxygen are analyzed.

\section{Introduction}

It is desirable if we can achieve fundamental understanding of the three-phase system consisting of carbon support, polymeric ionomer, and Pt catalyst nanoparticle, where the most essential electrochemical events for fuel cell operation take place in the presence of other molecular species such as water, proton, and oxygen. Although variety of cuttingedge in-situ and time-resolved probing techniques would be available to investigate nanometer-scale systems, the structure and dynamic behavior of the three-phase system of fuel cell has not been thoroughly analyzed at molecular level, which is mainly due to such complicated multi-component feature of the three-phase system in several tens of nanometers. In this context, we have simulated the three-phase systems using multi-scale first-principles modeling approach consisting of quantum mechanical density functional theory (DFT) and molecular dynamics (MD) simulations in order to investigate the nanophase-segregation of polymeric ionomers and water molecules, surrounding $\mathrm{Pt}$ nanoparticle on graphitic carbon support. For this, we developed a force field based on DFT computations and ran large-scale MD simulations. Another topic we would like to address is the Pt dissolution that degrades the fuel cell performance by decreasing the active catalyst surface area. In order to elucidate the Pt dissolution mechanisms under a certain surface potential condition, we implemented the DFT computation of various charged Pt surface in the presence of water.

Modeling of Three-Phase System. To achieve high quality results from the three-phase system, first, we built probable structures consisting of all the components in the simulated three-phase system. These are structures of the various moieties in the fuel cell (ionomer segments, water molecules, hydronium, and the oxygen molecule). Using DFT simulations we prepared an accurate force field describing the molecular interactions between the components. To ensure that we are correctly describing the long range 
interactions, we used the M06 functional (1). Figure 1 shows an example for the water-Pt nanoparticle pair. The binding energy was defined as :

$$
\Delta E_{\text {binding }}=E_{\text {total }}-\sum_{i} E_{\text {component }, i}
$$

where $E_{\text {total }}$ and $E_{\text {component }}$ are the total energy of the system and the energy of each component, respectively.

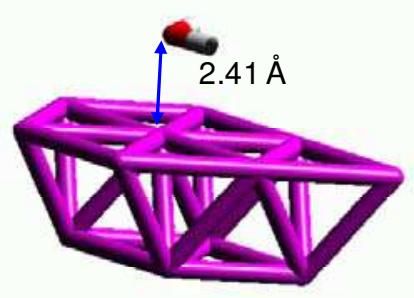

(a)

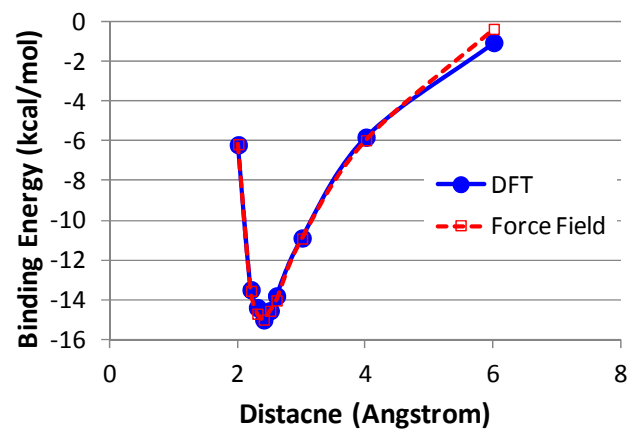

(b)

Figure 1. Change of binding energy as a function of distance between water and $\mathrm{Pt}$ model nanoparticle: (a) model system; (b) binding energy curves. The red curve was calculated from the new force field developed from the DFT computation. This was done for all moieties on our system, on different catalyst surfaces.

Using our DFT derived forcefield, we build an atomistic level simulation of the three phase system, as shown in Figure 2. In the figure we see nanophase-segregation of the polymeric ionomers and water molecules around $\mathrm{Pt}$ nanoparticle. The water phase is essential to transport the proton.

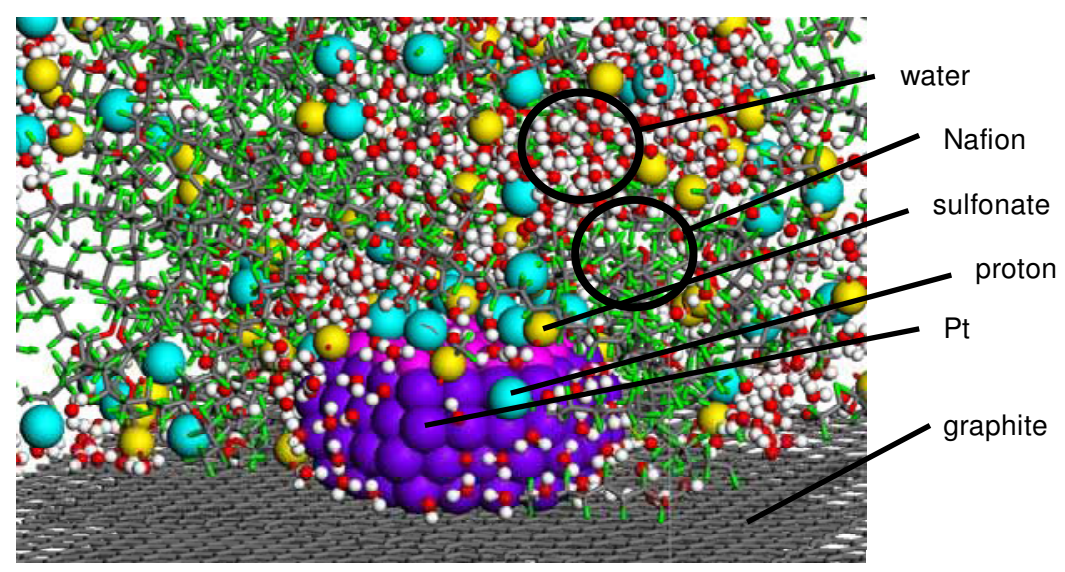

Figure 2. Three-phase system consists of Pt nanoparticle, polymeric ionomer, and carbon support with other molecular species such as water, proton and oxygen.

DFT Study on Pt Dissolution. Since it is known that Pt catalyst particles in cathode are dissolved under certain cathodic potentials, we investigated the electrochemical stability of Pt nanoparticle under various cathodic potentials using DFT computations with the double reference method suggested by Filhol and Neurock (2) and the nudged elastic 
band method $(3,4)$. As observed in Figure 2, the abundant of water molecules are adsorbed on the surface of Pt nanoparticle due to the favorable binding energy, and these water molecules are very essential to accommodate the dissolved Pt atoms.

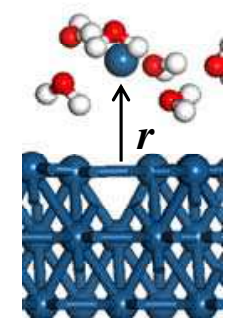

(a)

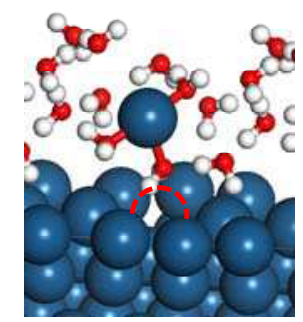

(b)

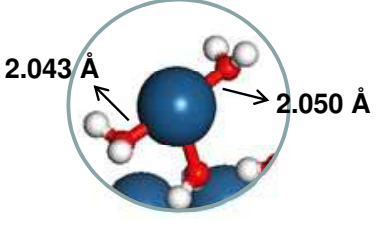

Figure 3. (a) Dissolution of Pt atom from Pt surface in the presence of water molecules; (b) association of Pt atom with water molecules.

\section{Results and Discussion}

Coverage of Platinum Nanoparticle. From the equilibrated structures of the three-phase system, we calculated the surface area various moieties occupied on the surface of the catalyst particle. To measure the surface area, we emplyed the Connolly surface method (5) with a probe radius of $1.4 \mathrm{~A}$ that is the radius of water molecule. Therefore, any feature on a surface smaller than this is not counted. Figure 4 shows an example of the surface area of Pt nanoparticle: the available surface area of Pt nanoparticle is calculated by subtracting the total surface area of Pt nanoparticle by the contacting area of $\mathrm{Pt}$ nanoparticle with graphite.
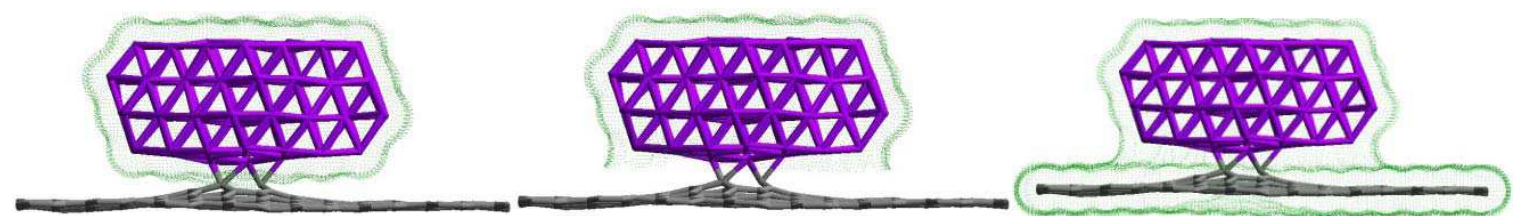

Figure 4. The Connolly surface wraps the selected atoms using a probe sphere. A) the platinum particle is wrapped b) the part of the platinum particle not contacting the graphite is wrapped c) all atoms are wrapped. By subtracting b) from a) we can calculate the contact patch of the platinum particle on the graphite surface.

The coverage for various moieties on the platinum surface is summarized in Figure 5, showing the surface area occupied by each chemical species. The active area of the catalyst is defined as the area occupied by water and hydronium, while the inactive area is the area occupied by the Nafion polymer. As we can see in the graphs, the active area is approximately $60 \%$ of the total area. In particular we note that the $\mathrm{SO}_{3}^{-}$group occupies a significant amount of area, in agreement with experimental data (6). 

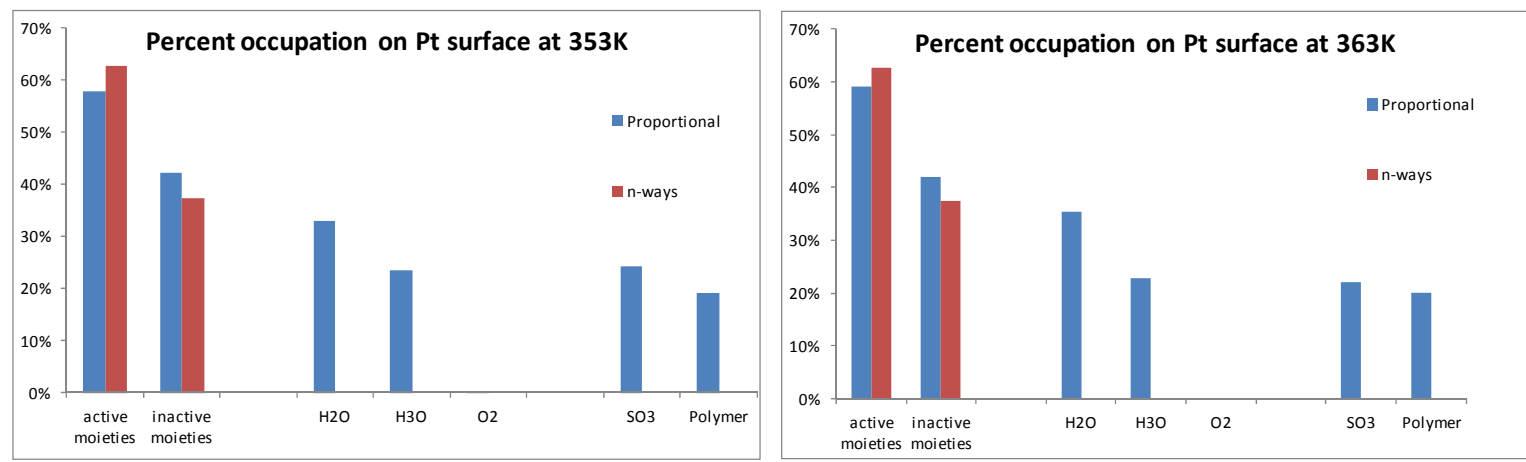

Figure 5. Coverage of various species on bare platinum at $353 \mathrm{~K}$ (left) and $363 \mathrm{~K}$ (right).

Distribution of Oxygen Molecules. Figure 6 shows the radial distribution functions (RDF) for oxygen surrounded by water (a) and fluorine in Nafion backbone (b). , indicating that oxygen molecules have a clear preference to be near the fluorinated phase and not in the water phase of the Nafion electrolyte. We think this is because the water phase prefers to maintain a strong hydrogen bond network. Dissolving oxygen molecules in water phase would disrupt the hydrogen bond network. In contrast, the fluorine atoms of Nafion are not as strongly affected by the oxygen molecules. Therefore, the oxygen molecules can be distributed in the Nafion phase more easily.

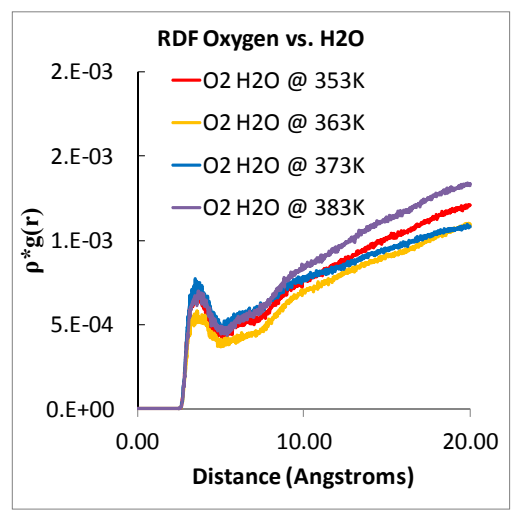

(a)

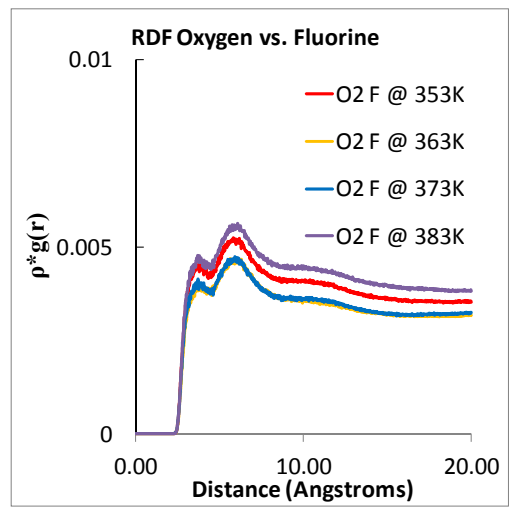

(b)

Figure 6. Radial Distribution Function (RDF) for oxygen - water pair (a) and for oxygen- fluorine pair (b). Notice that there is a clear preference for the oxygen molecules to be in the fluorinated phase.

From previous studies of Henry's law coefficient of oxygen molecule for hydrated Nafion membrane, we note that there is a large variation in the reported values of Henry's Law coefficient (7), which might be due to the various kinetic factors and processing history for sample preparation. If we assume that the volume fraction between these experiments is constant for a given water content, we found that our simulation results are consistent with the experimental Henry's law coefficient, indicating that oxygen molecules reside in the Nafion phase, but can also be found on the Nafion water interface. 
Dissolution of Pt. The nudged elastic band (NEB) is a method for finding saddle points and minimum energy paths between known reactants and products by optimizing intermediate images along the reaction path. The optimization is done by adding spring forces along the band between images and by projecting out the component of the force due to the potential perpendicular to the band. For the Pt dissolution study, the energy minimized initial and final states were prepared for applied three different electrode potentials $(-1.76 \mathrm{~V}, 0.41 \mathrm{~V}$ and $2.16 \mathrm{~V})$ calculated using the double reference method. Applied electrode potentials are one of the most important factors influencing the dissolution of Pt atom. Water molecules and/or hydroxides are favorably bonded to the dissociated Pt atom as the potential increases, which may lead to the more stabilized dissolution state (Figure 7a).

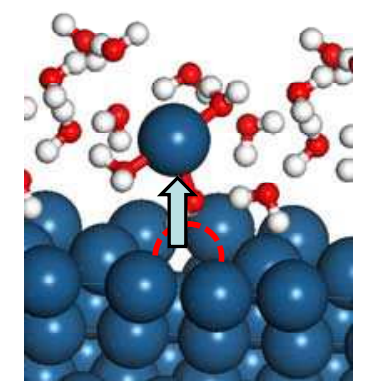

(a)

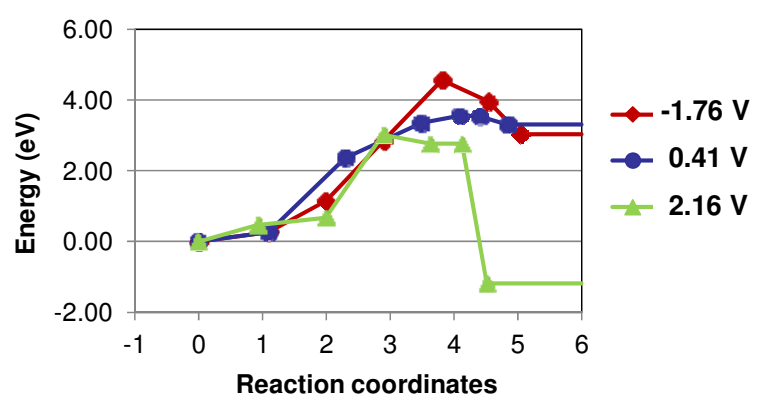

(b)

Figure 7. Reaction coordinates (a) is measured from the initial position to the position of dissociated Pt atom. Free energy changes are plotted at (b) as a function of reaction coordinates.

The energy differences between initial and final states were calculated to be $3.034 \mathrm{eV}$, $3.310 \mathrm{eV}$, and $-1.186 \mathrm{eV}$ for $-1.76 \mathrm{~V}, 0.41 \mathrm{~V}$ and $2.16 \mathrm{~V}$, respectively. At $-1.76 \mathrm{~V}$ and $0.41 \mathrm{~V}$, initial energy states are more stable than final states but $1.62 \mathrm{~V}$, where final state has lower energy than initial state. Transition state and activation energy barrier can be found by the NEB method. Reaction coordinates is a function of distance (r), where the distance is measured from the initial position of dissociated Pt atom to the final position. Figure $7 \mathrm{~b}$ illustrates the change of energy as a function of reaction coordinates. The height of energy barrier, as shown in Figure $7 b$, decreases as the electrode potential increases. It implies that the dissolution occurs more favorably at higher reduction potential (lower electrochemical potential).

\section{Conclusions}

By performing DFT modeling, we developed force fields describing the interaction of many molecular species with the surface of Pt nanoparticle. We performed MD simulations of three-phase systems using these newly developed force fields and analyzed the nanophase-segregated structures around the Pt nanoparticle. It is found that the surface of Pt nanoparticle is occupied by water, hydronium and sulfonate of Nafion. Thus, around $60 \%$ of the surface of Pt nanoparticle is available in the three-phase system. We also analyzed the distribution of oxygen molecules in three-phase system using the radial distribution function. In equilibrium state, the oxygen molecules have clear preference for Nafion phase, which is in agreement with the experimental observation. 
We investigated the dissolution of $\mathrm{Pt}$ in the presence of water phase using the double reference method and the nudged elastic band method within DFT modeling. We find the energy barrier for $\mathrm{Pt}$ dissolution is decreased with increasing reduction potential (decreasing electrochemical potential).

\section{Acknowledgement.}

This research was supported by Ballard via the Department of Energy (DOE) grant No. DE-EE0000466.

\section{References}

(1) Y. Zhao and D. G. Truhlar, Chemical Physics Letters, 502, 1 (2011).

(2) J.S Filhol, M.Neurock, Angew. Chem. Int. Ed., 45, 402 (2006).

(3) D. Sheppard, R. Terrell, and G. Henkelman Optimization methods for finding minimum energy paths, J. Chem. Phys., 128, 134106 (2008).

(4) G. Henkelman, B.P. Uberuaga, and H. Jónsson, A climbing image nudged elastic band method for finding saddle points and minimum energy paths, J. Chem. Phys., 113, 9901 (2000).

(5) M. L. Connolly, Science, 221, 709 (1983).

(6) R. Subbaraman, D. Strmcnik, V. Stamenkovic and N. M. Markovic, J. Phys. Chem. C, 114, 8414 (2010).

(7) R. F. Mann, J. C. Amphlett, B. A. Peppley and C. P. Thurgood, J. Power Sources, 161, 775 (2006). 